\title{
e-Phaïstos
}

e-Phaïstos

Revue d'histoire des techniques / Journal of the history

of technology

IX-1 | 2021

Autour de Léonard de Vinci

\section{Le verre mousseline : une innovation industrielle dans la France du XIX ${ }^{\mathrm{e}}$ siècle}

Muslin Glass: a French Industrialised Innovation in 19th Century

\section{Fabiola Lozano}

\section{OpenEdition}

Journals

Édition électronique

URL : https://journals.openedition.org/ephaistos/9033

DOI : 10.4000/ephaistos.9033

ISSN : 2552-0741

Éditeur

IHMC - Institut d'histoire moderne et contemporaine (UMR 8066)

Référence électronique

Fabiola Lozano, "Le verre mousseline : une innovation industrielle dans la France du XIXe siècle », ePhaïstos [En ligne], IX-1 | 2021, mis en ligne le 27 avril 2021, consulté le 17 septembre 2021. URL: http://journals.openedition.org/ephaistos/9033 ; DOI : https://doi.org/10.4000/ephaistos.9033

Ce document a été généré automatiquement le 17 septembre 2021.

Tous droits réservés 


\title{
Le verre mousseline : une innovation industrielle dans la France du XIX ${ }^{\mathrm{e}}$ siècle
}

\author{
Muslin Glass: a French Industrialised Innovation in 19th Century
}

Fabiola Lozano

1 Le problème qui se pose dans les différents procédés de décoration sur verre, à chaud ou à froid ${ }^{1}$, est la réalisation et la reproduction des dessins d'une bonne exécution ; soit par l'application des couleurs vitrifiables ou, dans le cas de la gravure, des substances grasses, afin de préserver les parties qui ne doivent pas être exposées à l'acide. De ce fait, à partir des années 1830, les procédés traditionnels d'impression à l'empreinte et au pochoir se perfectionnent et s'industrialisent. Cela se traduit par une amélioration de la qualité, une augmentation de la production et une réduction des coûts de fabrication. On applique ces techniques notamment dans la fabrication du verre mousseline, mais aussi dans la réalisation des décors des fonds et des frises des vitraux, réduisant également leur prix.

2 L'impression au pochoir est la première à être appliquée mais aussi la plus employée dans l'exécution du verre mousseline. Bien que cette méthode semble remonter au $\mathrm{XV}^{\mathrm{e}}$ siècle, ce n'est qu'au XIX ${ }^{e}$ qu'elle devient courante. Le procédé, copié de l'impression sur tissus, utilise le principe des caches pour laisser passer l'encre seulement aux endroits qui doivent être imprimés. Les pochoirs, faits de matériaux divers tels que papier, carton ou métal, sont positifs ou négatifs. Ainsi, soit on dispose le pochoir sur le verre et on applique la peinture dans les entailles, soit on recouvre le verre d'une épaisse couche de peinture et on fait les enlevages à travers les jours du pochoir par grattage, à l'aide d'une brosse dure. 


\section{Le procédé à chaud}

\section{L'application de matières vitrifiables}

3 La première méthode de dépolissage du verre que les artisans du verre mousseline développent consiste dans l'application d'un émail blanc opaque à base de plomb et d'étain, qui se fixe à la surface du verre à une température à laquelle celui-ci est seulement ramolli (Peligot 1877: 190-192). Le pionnier de ce procédé est l'artiste peintre Charles Duval, comme on peut le constater dans le rapport de l'Exposition Nationale de 1849 :

\footnotetext{
«M. Charles Duval est inventeur d'un procédé d'impression et d'application de couleurs vitrifiables sur verre blanc: La feuille de verre blanc est recouverte d'une feuille de plomb gravée, et l'on saupoudre le tout de poussière de couleurs vitrifiables mêlées avec une petite quantité de gomme, également en poudre, pour donner à la matière colorante la faculté de rester adhérente au verre légèrement humecté. Si l'on veut appliquer d'autres couleurs, sur d'autres parties du dessin, on fait usage d'une nouvelle feuille de plomb convenablement gravée. La feuille de verre ainsi colorée est passée ensuite à la moufle comme à l'ordinaire » (Ebelmen 1850 : 883).
}

Il enregistre un premier brevet relatif à cette méthode en 1837: "Application de substances blanches et colorantes sur le verre et autres corps solides par des procédés extrêmement rapides et dont les résultats pourront être employés à la décoration de toute espèce d'édifice $»^{2}$. Les couleurs réduites en poudre fine sont appliquées sur le verre préalablement gommé. Pour assurer une couche de couleur uniforme, on place les feuilles de verre dans une boîte, puis on applique les couleurs à l'aide d'un tamis ou d'un soufflet. L'année suivante, Duval cède les droits de son invention à l'émailleur Jean Pierre Marrel, qui introduit comme nouveauté l'emploi d'étoffes telles que dentelles et tulles montées sur des cadres, en tant que pochoirs ${ }^{3}$.

En 1848, Duval dépose un nouveau brevet qui fait suite à celui de 1837 : «Procédés de peinture sur verre $»^{4}$. Le principe est le même avec quelques améliorations. Les poncifs, faits en feuilles de plomb ou de métal battu, sont galvanisés pour leur donner plus de rigidité. Les jours du poncif sont dessinés ou décalqués, puis enlevés à la pointe du burin. Chaque couleur qui comporte le sujet requiert un premier poncif pour le trait, un deuxième pour les demi-teintes et un troisième pour les ombres. Une fois la couleur réduite en poudre impalpable et répandue dans les jours du pochoir, Duval assure une fixation provisoire des couleurs en introduisant le verre dans une chambre à vapeur. La condensation de l'eau humidifie la peinture qui, en séchant, s'attache au verre. Ceci permet d'appliquer autant de couleurs que l'on veut et de les faire cuire toutes dans la même cuisson. Duval cède à nouveau les droits de son invention, cette fois-ci au peintre-verrier Pailleux qui introduit certaines modifications au procédé, dont une boîte pour l'application des couleurs qui dose la poudre au moyen d'une trémie à laquelle on adapte une soufflerie (fig.1). C'est pourquoi il sollicite un certificat d'addition et de perfectionnement en $1852^{5}$. 
Fig.1. Boîte pour l'application uniforme de couleurs en poudre sur les feuilles de verre lors de la fabrication du verre mousseline 

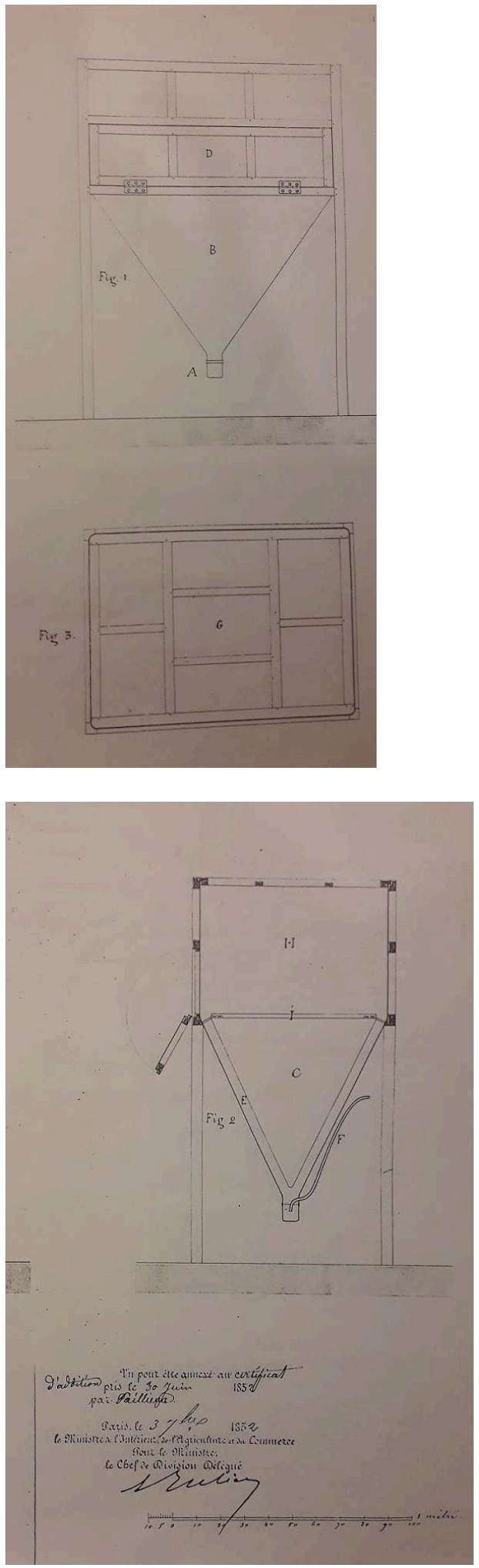
Explication du schéma annexé au brevet, fait par Pailleux :

A. Godet renfermant la poussière.

B. Pyramide en zinc dont la concavité est destinée à recevoir la pyramide $\mathrm{C}$ dont les plans laissent partout un intervalle d'environ quatre centimètres. Cet intervalle permet le passage de la poussière. C. Pyramide entrant dans la concavité de la pyramide B et superposée sur des pivots placés à ses quatre angles.

D. Porte à charnières garnie de zinc par laquelle on entre le verre.

E. Vide ménagé entre les deux pyramides pour le passage de la poussière.

F. Tube en plomb auquel s'adapte un boyau communiquant à une soufflerie pour soulever la poussière.

G. Châssis garni de toile et servant de couverture à la boîte.

$H$. Vide de la boîte dans lequel montent les nuages de poussière soulevés par l'effet de la soufflerie.

I. Base de la pyramide B servant de support aux feuilles de verre.

INPI, Certificat d'addition de Paillieux au brevet d'invention de Duval, déposé le 30 juin 1852

6 Le peintre Joseph Dumas, associé au chimiste Émile Godard, développe le procédé d'impression au pochoir par enlevage qui fait l'objet d'un brevet déposé en 1841 : "Moyen de vitrification propre à imiter la mousseline brodée, sur le verre à vitre ordinaire ${ }^{6}$. Leur méthode d'impression diffère de celle de Duval essentiellement dans le mode d'étaler les couleurs qu'ils appliquent en dissolution et non en poudre. D'ailleurs l'originalité de cette invention réside justement dans la préparation des deux compositions qu'ils emploient pour " mousseliner » les vitres. La première, destinée à réaliser tous les décors, est composée de sable blanc, potasse caustique, verre de borax, calcin de cristal, minium purifié, oxyde blanc d'étain et peroxyde de manganèse. La deuxième, qui doit être plus diluée donc plus transparente, est destinée à donner la teinte générale qui sert de fond. Celle-ci comporte presque les mêmes composants à l'exception de l'oxyde blanc d'étain et du peroxyde de manganèse. À leur place, ils ajoutent de l'oxyde du cobalt, afin de donner aux vitres la teinte bleutée des mousselines. Une couche de la première composition, délayée dans une dissolution de gomme arabique, est étendue de façon uniforme sur la vitre. Lorsque les couleurs ont bien séché, ils posent les pochoirs par-dessus et, à l'aide d'une brosse à poils de sanglier, ils enlèvent la couleur qui est restée à découvert, restituant la transparence à toutes les parties qui doivent servir de fond. Puis une couche de la deuxième composition, délayée dans l'essence de térébenthine, est appliquée avant de sécher comme la première. Après quoi les vitres émaillées passent à la cuisson.

7 Ce procédé d'impression par enlevage présente toutefois l'inconvénient de répandre les poussières des couleurs enlevées par la brosse dans l'air. Celles-ci sont nocives en raison de l'oxyde de plomb qu'elles contiennent et rendent cette opération dangereuse pour la santé des ouvriers. Le chimiste Eugène Péligot (1811-1890) remarque la nocivité de cette méthode et conseille de réaliser cette opération sous la hotte d'une cheminée ayant un fort tirage, dans une salle bien aérée. Il insiste même sur l'usage d'un masque de protection:

«Il serait à désirer qu'on fasse usage du masque inventé par M. Paris de Bourget ${ }^{7}$, et employé pour l'émaillage de la tôle. C'est une sorte de muselière en caoutchouc et en gutta-percha avec un appendice en toile métallique garni extérieurement d'une flanelle mouillée; ce tissu tamise l'air que l'ouvrier respire et en retient les poussières » (Peligot 1877).

8 Le chimiste et peintre-verrier messin Louis-Napoléon Gugnon (1808- ?), très investi dans le développement des méthodes d'impression sur verre et dans l'industrialisation 
du verre dépoli, dépose de nombreux brevets. Ceux qui datent de 1861, 1865 et 1866 concernent très précisément des "Perfectionnements apportés à la fabrication des verres mousseline $»^{8}$. Il débute l'an 1838 dans son atelier rue des Murs à Metz, associé à son beau-frère, le célèbre peintre-verrier Maréchal, puis, vers l'an 1853, ils transfèrent leur atelier à Paris (Barbé 1976 : 264). Ils rencontrent un grand succès mais, peu après, Gugnon et Maréchal décident de s'installer séparément. Auguste-Raphaël (1835- ?), fils de Gugnon, travaille à côté de son père. Pour certaines des inventions, il est impossible de préciser si l'auteur est le père ou le fils (fig.2). En ce qui concerne la fabrication des verres mousseline, Gugnon apporte notamment une amélioration à la méthode d'impression par enlevage. Étant donné que le pochoir n'a pas l'étendue de la feuille de verre, il faut le déplacer successivement, de manière à couvrir des dessins la totalité du verre. Il développe alors un appareil composé d'une table qui glisse horizontalement sur une armature en fonte, où l'on place la feuille de verre à plat ; et d'un châssis en métal pourvu d'une charnière, où l'on a fixé le pochoir. Ce châssis se positionne tour à tour sur la table que l'on fait glisser manuellement à l'aide d'une vis sans fin actionnée par un volant. Sur le côté, un cylindre pourvu de cannelures hélicoïdales, dont le pas correspond à la distance de progression de la table à chaque étape, assure la reproduction des dessins à l'endroit précis sur la feuille de verre (Peligot 1877).

\section{La cuisson du verre mousseline}

9 La fusion de l'émail se fait dans un four à moufle chauffé au bois, dans lequel on empile les feuilles émaillées les unes au-dessus des autres, en interposant entre elles une mince couche de plâtre en poudre, dans le but d'empêcher les feuilles de se souder entre elles lors de la cuisson. L'ouvrier doit surveiller la cuisson soigneusement et de façon à entretenir une température uniforme à l'intérieur du moufle. Si la température du fourneau est trop élevée, le plâtre risque de laisser des traces sur l'émail et faire fondre le verre lui-même. Par contre, si la température est trop basse, l'émail n'adhère pas au verre (Peligot 1877).

10 Face à ces contraintes, Gugnon développe un four de cuisson pour verres émaillés, inspiré du système de four à refroidir le verre à vitre en continu du verrier Bievez ${ }^{9}$. Le fait de cuire les feuilles de verre en continu évite de les superposer, ce qui supprime la couche de plâtre entre les feuilles et, par là même, le risque de traces sur l'émail. Gugnon se sert alors d'un four dans lequel la feuille de verre avance lentement, en s'échauffant graduellement jusqu'à la chambre de cuisson. Là, elle est placée sur une pierre mobile qui entre dans l'arche chauffée au rouge. L'ouvrier suit le progrès de la vitrification dans la paroi du four : une fois la fusion de l'émail achevée, la pierre sort de l'arche. Lorsque la feuille devient assez rigide pour être enlevée sans se déformer, il la fait passer dans la galerie à recuire où elle refroidit lentement. L'ouvrier la retire à l'extrémité de cette galerie (Peligot 1877). Gugnon dépose deux brevets de fourneaux pour la cuisson du verre émaillé : le premier en 1865, spécifiquement conçu pour la cuisson du verre mousseline et l'autre, en 1870, développé pour l'étendage et le bombage du verre à vitre ainsi que pour la cuisson de verres ornementés ${ }^{10}$ 


\title{
Les procédés à froid
}

\section{La gravure à l'acide}

\section{Le procédé Gugnon}

Dans les années 1850, l'acide fluorhydrique se révèle un moyen efficace pour graver le verre et devient une nouvelle ressource pour la fabrication du verre mousseline. Le peintre-verrier Gugnon, un des précurseurs de la gravure à l'acide, développe aussi la fabrication du verre mousseline par ce moyen et dépose plusieurs brevets à ce sujet au cours des années 1850. Deux de ses inventions recréent, à l'aide de l'acide fluorhydrique, les méthodes d'impression que nous venons d'exposer. Le procédé breveté en $1853^{11}$ s'inspire de celui développé par Charles Duval, mais au lieu de couleurs vitrifiables, Gugnon épand sur le verre du bitume pulvérisé. Le bitume est la substance qui préserve de l'acide les parties du verre qui ne doivent pas être gravées. Selon Gugnon, l'avantage de son procédé consiste à remplacer le bitume liquide appliqué au pinceau dans le procédé de gravure usité, par le bitume sec, réduit en poudre, appliqué à travers un tamis ou par un moyen mécanique. On applique sur la feuille de verre une légère couche d'un corps gras, par exemple de l'essence de térébenthine, on pose le pochoir que l'on a préparé d'avance sur cette couche encore fraîche et on agite au-dessus un tamis contenant de la poudre de bitume. L'apport de Gugnon à ce procédé consiste en un engin qu'il conçoit pour répandre les poudres d'une façon plus rapide et plus régulière, et qu'il décrit ainsi :

\begin{abstract}
«Comme moyen plus précis et plus rapide d'exécution j'ai remplacé le tamis par une boîte demi-cylindrique à sa base, haute de deux mètres, longue de trois mètres \& large d'un mètre. À la partie demicylindrique et dans le sens long de la boîte j'ai placé un arbre armé d'ailes, comme celles d'une machine à vanner le grain. Je place mes poudres préparées dans cette boîte, \&, à l'aide d'une manivelle adaptée à l'arbre en dehors de la boîte, je mets cet arbre en mouvement, \& les poudres se trouvent agitées par les ailes fixées à l'arbre. Alors je glisse promptement, par une ouverture réservée, sur deux coulisses intérieures, une tablette sur laquelle j'ai préparé à l'avance mon dessin sur une glace couchée d'essence. La boîte étant bien close, la poudre qui est en suspension retombe également sur tous les dessins que la table contient $»^{12}$.
\end{abstract}

Une fois les dessins reproduits ainsi sur le verre, il les entoure d'un bourrelet de cire molle préparée à cet effet. Puis, il verse l'acide fluorhydrique étendu d'un tiers d'eau sur la feuille de verre. Il laisse agir environ une quarantaine de minutes et le travail est fini. D’après Gugnon, «ce procédé permet de produire mécaniquement plus que l'homme le plus exercé ne peut faire en un mois, car deux ouvriers peuvent graver environ 20 mètres superficiels par jour » (Gugnon 1859 : 524-525).

Un brevet déposé par Gugnon en $1855^{13}$ reprend deux procédés d'impression existants qu'il applique également à la gravure sur verre. Le premier correspond à l'impression par enlevage de Dumas et Godard pendant que le second imite le système d'impression et de décalquage employé dans les faïenceries. Ces procédés étant déjà assez connus, le problème qui se pose à Gugnon, dans le cas particulier de la gravure sur verre, consiste 
à trouver les substances grasses et résineuses appropriées pour composer des réserves qui adhèrent bien au verre et supportent l'action de l'acide fluorhydrique pendant des heures. Dans le cas de l'impression par enlevage, qui nécessite accessoirement que les réserves puissent être appliquées en couches minces et uniformes et retirées facilement par frottement à l'aide d'une brosse, il opte pour un composé d'asphalte ou bitume de Judée et d'élémi mou fondus ensemble. Après refroidissement, ce mélange est réduit en poudre fine et dilué avec une légère proportion d'eau de savon.

Quant à l'impression par décalquage, le dessin peut s'exécuter sur bois, en taille douce ou autrement, en fonction de la nature ou de la finesse du travail que l'on veut faire. L'impression se réalise comme à l'ordinaire, l'encre seule varie. Étant destinée à former réserve sur le verre, elle doit posséder les mêmes propriétés que celles décrites plus haut, avec toutefois une consistance plus dense pour le tirage. Gugnon emploie une pâte à base d'asphalte fondu dans de l'essence de térébenthine, à laquelle il donne la consistance voulue en ajoutant de l'huile grasse. Le transport du dessin se fait sur un papier qui est préalablement préparé avec une couche d'eau gommée. Au fur et à mesure du tirage, on mouille l'épreuve qui est appliquée sur le verre en imprimant une légère pression avec un tampon doux. L'encre se colle au verre et le papier, dont l'humidité a détrempé la colle, s'enlève de lui-même. Comme dans les autres procédés, la pièce est ensuite légèrement chauffée, pour bien faire adhérer l'encre.

L'année suivante, un certificat d'addition joint à ce brevet porte sur un mode d'impression par décalquage analogue à celui que l'on vient d'énoncer, où l'on se sert de gravures dont le motif est creusé plus profondément et sur lesquelles on étale une encre beaucoup plus épaisse. À l'aide d'une racle en acier, on enlève le surplus de l'encre et on obtient ainsi des dessins à contours très nets, sur des verres doublés ou sur des verres blancs, mais aussi des gravures en creux brillantes ou mates ${ }^{14}$. Gugnon enregistre encore des brevets relatifs à la gravure où les principes sont à peu près les mêmes que ceux déjà décrits avec quelques modifications.

\section{La méthode Aubriot, fruit de la collaboration avec Gugnon}

Édouard Aubriot collabore aux inventions de l'atelier Gugnon et fils (fig.3). On ne peut pas préciser la date où il intègre l'atelier. Toutefois, il épouse Joséphine-Claire Gugnon, fille de Louis-Napoléon Gugnon, en $1860^{15}$. Aussi son brevet relatif à la gravure sur verre de 1863 est déposé au nom d'« Aubriot Édouard dit Aubriot-Gugnon $»^{16}$. En outre, il fait enregistrer deux brevets en 1866 et 1874, ceux-ci uniquement au nom d'Aubriot: «Perfectionnements ayant pour effet de donner aux verres-mousseline l'apparence de verres gravés" et "Moyens de fabrication des verres à dessin-tulle avec teintes colorées multiples $»^{17}$. 
Fig.2. L'Atelier de peinture sur verre Gugnon \& Fils, spécialistes du verre mousseline

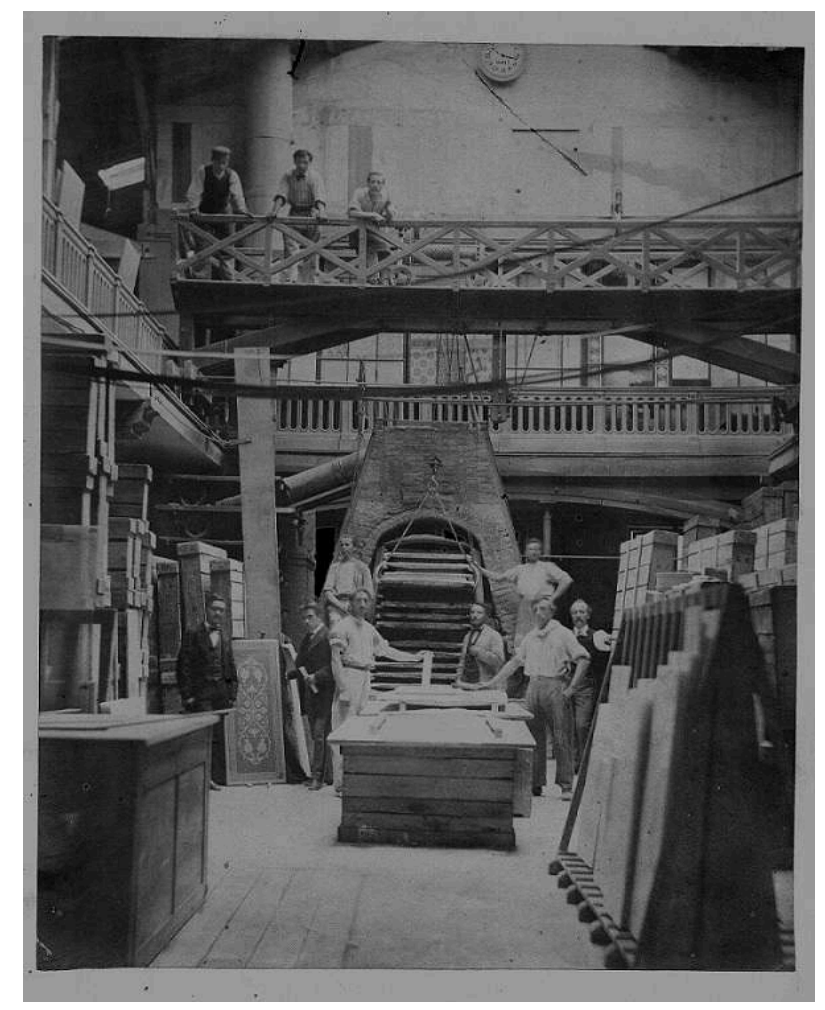

L'atelier était situé au 130 Rue du Faubourg St-Denis à Paris, vers les années 1870-1890.

(c) Christian Fournié

17 Louis-Alphonse Salvetat (1820-1882) fait une description exhaustive de la méthode Aubriot dans son rapport de 1877 (Salvetat 1877 : 621-626). Le système d'exécution de verres mousseline d'Aubriot rassemble la plupart des techniques que nous venons d'exposer. Après avoir nettoyé parfaitement la surface du verre, on étend une première couche de peinture mélangée à un peu d'eau gommée que l'on égalise dans les deux sens au blaireau. Une fois la peinture bien sèche, on applique le pochoir par-dessus et on frotte avec une brosse dure pour enlever la couleur des parties qui doivent être transparentes. Le pochoir est déplacé au moyen de repères sur toute la surface du verre, de façon à imprimer les dessins dans toute la feuille. On applique alors sur le verre un châssis où l'on a monté soit un tulle, soit une mousseline, soit une dentelle, puis on passe le tout dans la boîte à poussière. Cette boîte hermétique renferme, dans sa partie inférieure, un réservoir rempli d'une couleur blanche pulvérisée. Au moyen d'un soufflet, on répand uniformément la couleur sur la feuille de verre qui a été glissée au fond de la boîte par une trappe. Lorsque le châssis est enlevé, on peut apprécier les dessins du tissu imprimés sur le verre. La couleur est ensuite fixée dans une chambre à vapeur. Finalement, on peut passer le verre au four pour faire adhérer la couleur ou bien le graver à l'acide.

\section{Le dépolissage mécanique}

18 Les vieilles techniques de dépolissage mécanique consistent essentiellement à frotter le verre avec des matières abrasives telles que terres ou émeris. Leur origine est ancienne et elles évoluent plus lentement, en raison de l'effort physique qu'elles demandent. 
Nous avons repéré un brevet relatif à cette méthode, enregistré en 1808 par le dépolisseur de verre Dupieu (le métier de dépolisseur existe donc au début du XIX siècle). Il s'agit d'un brevet de cinq ans qu'il a renouvelé à son expiration, en 1813. La notice énonce un « procédé propre à dépolir les globes et garde-vues en verre et cristal, à l'usage de toutes sortes de lampes ». Voici son mémoire descriptif :

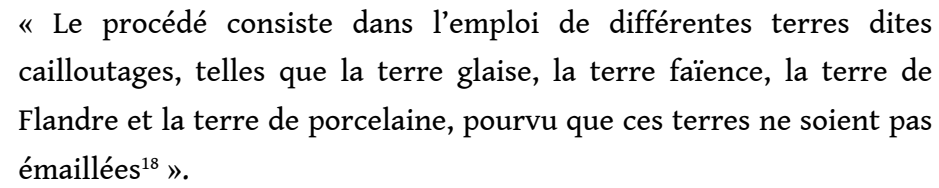

Le dépolissage est souvent utilisé dans les verres de luminaire, car il permet d'adoucir leur luminosité. Jean-François Vernert fait breveter en 1817 un procédé similaire à celui de Dupieu: «Globes et garde-vues en verre ou en cristal dépoli, représentant toutes sortes de sujets en peinture, et à l'usage des lampes à courant d'air sans exception ${ }^{19}$ ». Pour dépolir, Vernert emploi de l'émeri tandis que, d'après lui, à l'époque, on n'employait ordinairement que du grès, méthode moins expéditive. Son brevet ne porte pas simplement sur le dépolissage, mais aussi sur l'idée de peindre, à la manière des peintres sur porcelaine, les globes et garde-vues, une fois dépolis, puis de fixer la peinture par une cuisson, comme dans les procédés analysés précédemment.

François Fournier, graveur sur cristaux, conçoit la première machine à dépolir le verre à vitre. C'est pourquoi il dépose un brevet en $1846^{20}$. Cette machine se compose d'une caisse qui comporte un certain nombre de tiroirs rangés verticalement. Les fonds de tiroirs sont garnis d'une étoffe en laine, qui sert à matelasser la feuille de verre logée à l'intérieur, pour en éviter la fracture. Les feuilles de verre sont retenues par des rebords en bois, de la même épaisseur que le verre, de manière à prévenir toute sorte de mouvement. Ces rebords contiennent également les matières abrasives qui servent au dépolissage. Fournier emploie à cette fin du sable siliceux d'une grosseur qui varie en fonction du dépoli à réaliser. Le sable doit être imbibé d'eau pendant l'opération. Les feuilles de verre sont couvertes d'un grand nombre de disques en fer, de deux centimètres de diamètre sur cinq à six millimètres d'épaisseur. Ces disques, livrés à eux-mêmes, agissent comme des petites molettes, actionnés par le mouvement d'oscillation général, qui est transmis à cette machine par un moteur quelconque (fig. 4). On peut se demander si ce système, qui semble très encombrant et plutôt compliqué à mettre en place, a continué à être appliqué. En effet, en 1868, Gugnon brevète à son tour une "Machine servant à faire des verres dépolis ou gravés en plein ou avec dessins, remplaçant ainsi les verres-mousseline et évitant leurs défauts habituels et les inconvénients que présente leur fabrication $»^{21}$, brevet qui reste à examiner. 


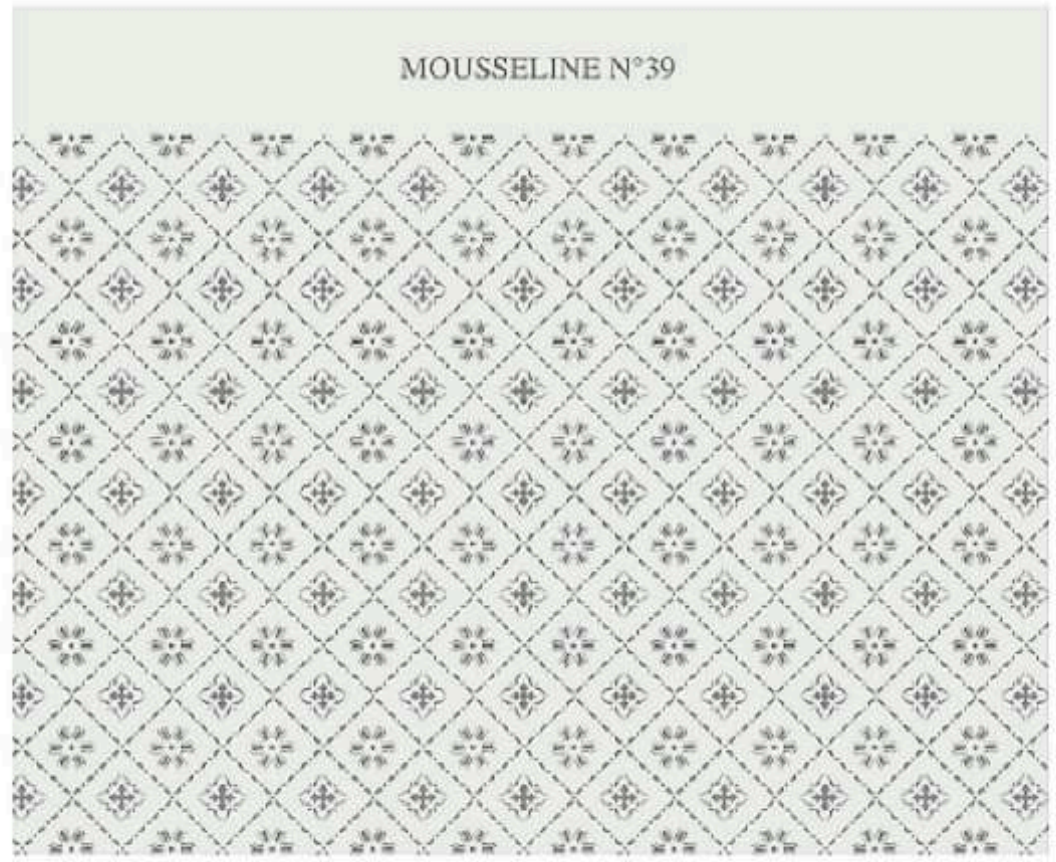

(c) Christian Fournié

\section{La gravure au jet de sable} un procédé mécanique qui consiste à corroder le verre par la projection de sable sur sa surface au moyen d'un jet d'air ou de vapeur. Cette méthode permet de dépolir le verre, de le creuser et même de le percer, en fonction de la pression et du diamètre du jet, du calibre du grain de sable projeté et de la durée du sablage. Le jet de sable est employé spécialement dans l'ornementation du verre pour graver des motifs mats sur fond brillant ou vice versa, ainsi que pour graver des verres doublés. On peut aussi sculpter le verre en le creusant à des profondeurs différentes et avoir un effet "camée ». Tout comme dans les autres méthodes de gravure, on applique des réserves ou des caches faites de papier ou d'une matière élastique, afin de préserver les parties qui ne doivent pas être sablées.

Au début des années 1870, Benjamin Tilghman (1821-1901), de Philadelphie, conçoit la gravure au jet de sable. Diplômé de l'Université de Pennsylvanie en 1839, Tilghman suit une licence en droit, suivant le souhaitait de son père, études qu'il abandonne bientôt pour s'associer à son frère Richard dans une activité d'entrepreneur et d'inventeur. Au début de la guerre de Sécession, en 1861, Tilghman se porte volontaire et rejoint le vingt-sixième régiment de Pennsylvanie. Dans l'armée, il gravit rapidement les échelons et passe de capitaine à commandant au sein de son régiment. Pendant ce temps, il se tient à l'affût des possibilités de nouvelles inventions. C'est ainsi que lors d'une patrouille de routine, Tilghman observe la corrosion provoquée par les tempêtes de sable à l'extérieur de vieilles fenêtres. Les vitres prennent un aspect rugueux et si on 
place quelque chose devant, par exemple des persiennes, on peut voir sa forme gravée sur la fenêtre. Il se demande si ce fait qui se produit de façon aléatoire dans la nature peut être reproduit de manière contrôlée.

Tilghman y parvient. En 1870, il obtient aux États-Unis la patente US 108,408 (Cassidy) et, en France, le brevet cote 1BB9089422, relatif à une "méthode de couper, percer ou user les pierres, les métaux et d'autres substances, etc.» auquel il joint par la suite plusieurs certificats d'addition. Le procédé est décrit ainsi :

"Un jet de sable propulsé à grande vitesse par une vapeur ou un courant d'air est employé comme outil pour tailler la pierre et autres matériaux et à une plus faible vitesse pour meuler et ornementer la surface du verre » (fig.5).

Le brevet d'addition de 1872 concerne les divers emplois du jet de sable : «tailler, forer, dépolir, dresser, pulvériser et graver les pierres, les métaux, le verre, le bois et autres substances dures ou solides ; pour nettoyer et aplanir les surfaces des articles en métal fondu ou en métal battu et enlever les scories, les écailles et toutes autres incrustations ; pour préparer les métaux à l'étamage, à l'émaillage, ou à être couvert de substances métalliques ou autres ${ }^{23}$.

Fig.4. Reproduction du motif $n^{\circ} 39$ du catalogue de verre mousseline de l'atelier Gugnon et Fils

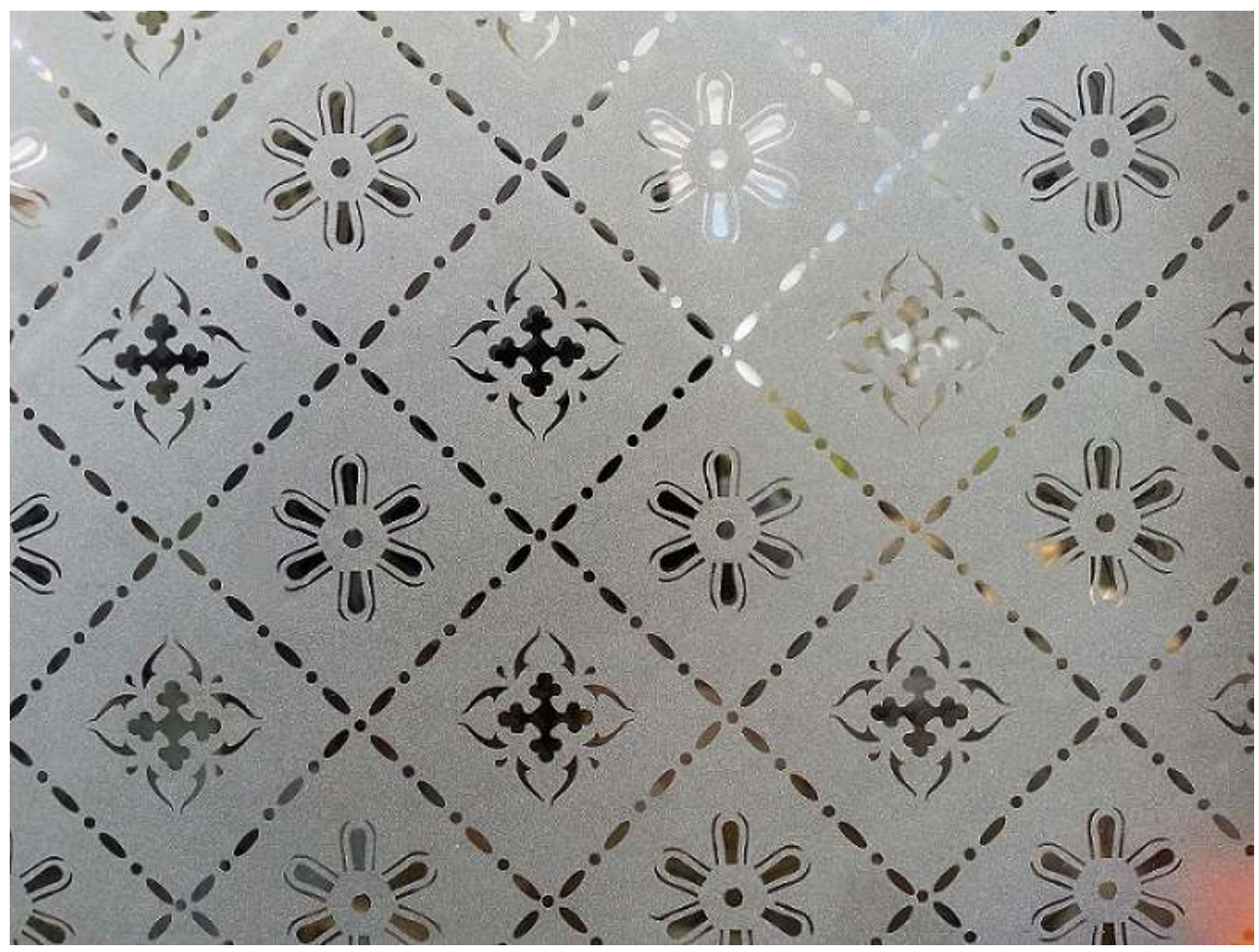

(c) Christian Fournié

En 1872, l'ingénieur Hervé Mangon (1821-1888) présente à la Société d'encouragement pour l'industrie nationale des échantillons de gravure sur verre, réalisés à l'aide d'un appareil qu'il a construit, inspiré du procédé Tilghman (Combes, Peligot $1872: 468-469$ ). Ce sont des plaques complètement dépolies ou gravées avec des dessins variés, ainsi 
que des éprouvettes et des flacons gradués. Péligot illustre et décrit la sableuse Mangon dans son ouvrage Le verre, son histoire, sa fabrication :

«[...] c'est une trémie contenant du sable bien sec A, qui s'écoule d'une manière continue par un tube $C$ dont on règle la longueur et l'inclinaison de manière à graduer à volonté la chute du sable : cet écoulement se fait par un tube étroit placé un peu au-dessous du tube qui amène le jet de vapeur ou le vent d'une machine soufflante. Des trous d'air comme dans les trompes [24], sont pratiqués à une petite distance du tube qui amène le vent. Le sable entrainé violemment par ce jet, est projeté avec force sur le corps E qu'on soumet à son action » (Peligot 1877 : 71-72) (fig.6).

Fig.5. Procédé Aubriot pour l'exécution des verres mousseline colorés

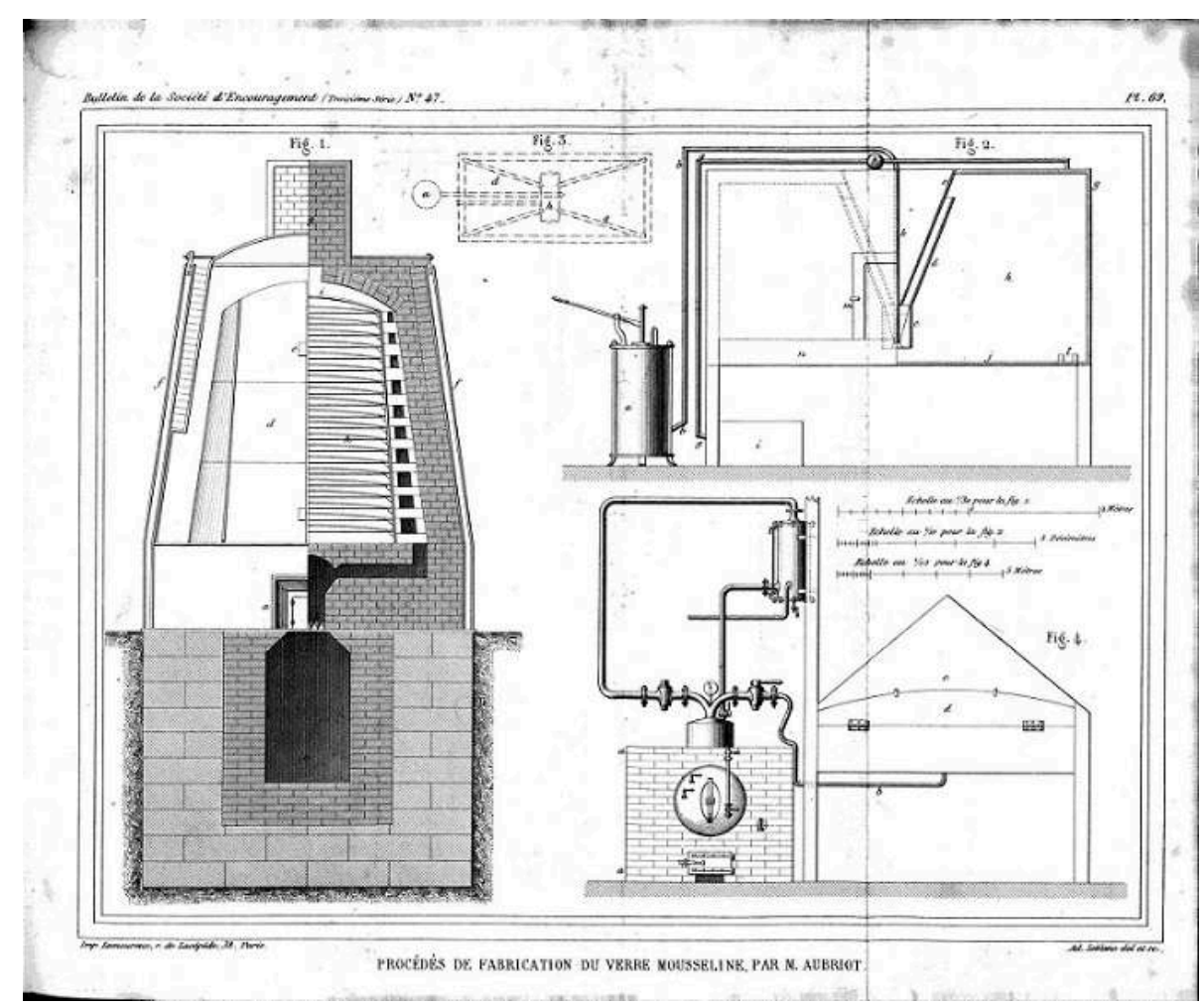

Moufle de cuisson (fig.1), Boîte à poussières (fig.2) et Boîte à vapeur (fig.3).

Bulletin de la Société d'Encouragement pour l'Industrie Nationale. Paris, 1877, 76e année. 3e série, tome 4, pl.69 - im.640, CNUM

Afin d'éviter les poussières pendant le sablage, il est conseillé d'enfermer l'appareil dans une cage vitrée. La technique du jet de sable permet de corroder rapidement diverses matières. Lors des expériences faites à New-York, avec une pression de 136 kilogrammes, un échantillon de corindon et une lime d'acier ont été percés en quelques minutes. Pour ronger le verre, on n'a certainement pas besoin d'une pression aussi forte. Selon Péligot, une lampe d'émailleur suffit. Cependant, la technique de gravure au jet de sable met plusieurs années à s'imposer, bien qu'à la fin des années 1870, l'idée est déjà répandue, comme on peut le constater dans le rapport de Clémandot relatif à l'Exposition de 1878 : 


\begin{abstract}
« Nous n'avons pu examiner les procédés de gravure et de polissage qui consistent dans un dépoli obtenu par un jet de sable lancé violemment sur le verre : c'est le procédé Stilgmann (sic), procédé très ingénieux et très original, comme tout ce que produisent les Américains. Nous ne le citons que pour mémoire, le jury ayant eu le regret de ne pouvoir en constater la valeur, puisque son inventeur ne l'a pas fait figurer à l'Exposition de 1878 »(Clémandot 1880 : 34-36).
\end{abstract}

En effet, bien que le principe soit ingénieux, la technique ne se répand que dans les années 1890 . Au cours des premières tentatives, le sable, projeté à l'époque par de la vapeur, devient rapidement humide, adhère et colmate les tuyauteries et oxyde rapidement les pièces. Dans les années 1880, Mathewson apporte quelques perfectionnements qui tendent à éliminer les inconvénients dus à l'utilisation de la vapeur comme fluide vecteur. Le procédé est quelque peu amélioré, mais son rendement reste faible ${ }^{25}$.

Fig.6. Machine à dépolir le verre à vitre

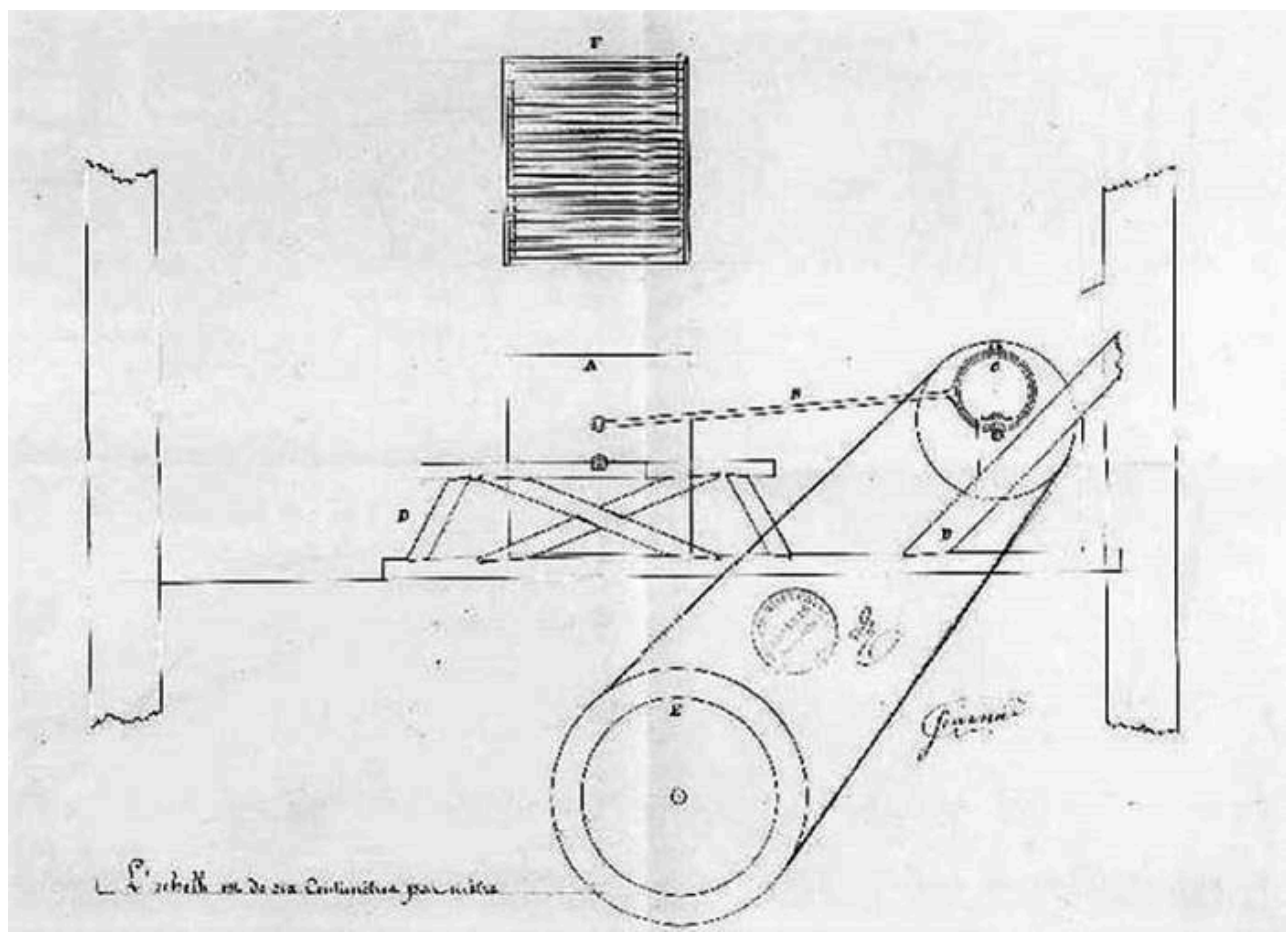

Les feuilles de verre rangées dans des tiroirs, sont dépolies à l'aide de matières abrasives par l'action de disques posés sur le verre, actionnés par le mouvement d'oscillation transmis par un moteur. INPI. Mémoire descriptif du brevet de François Fournier, déposé le 1er avril 1846, Cote 1BB3244.

En 1885, Tilghman invente le compresseur d'air qui permet de remplacer la vapeur par de l'air comprimé sec. Le sablage trouve un nouvel essor et son développement peut s'accélérer. Malgré tout, le rendement reste faible suscitant des recherches vers d'autres procédés, qui visent en particulier à en réduire les coûts. Quand, en 1886, Gutmann remarque l'importance du brevet Tilghman, il entreprend la construction de la première "machine à sabler par aspiration ", la plus ancienne connue en Europe. La technique du sablage proprement dite est née. En 1893, Gutmann obtient un brevet 
pour sa «sableuse à air comprimé » qu'il appelle sableuse à jet libre. Son procédé est toujours utiliséé ${ }^{6}$.

Fig.7. Sableuse Tilghman

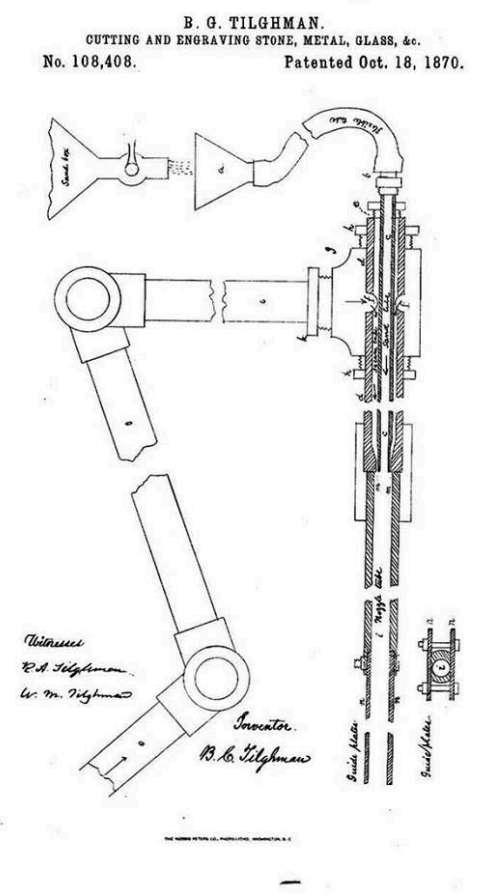

Ce fut la première machine à dépolir le verre par jet de sable.

Patent N¹08,408 du 18 octobre 1870. United States Patent and Trademark Office (USPTO)

La technique du sablage révolutionne la gravure sur verre et remplace graduellement, en grande partie, la gravure à la roue et à l'acide fluorhydrique. En 1925, Gaëtan Jeannin, de Boulogne-Billancourt, est le premier à présenter des verrières réalisées par cette technique à l'Exposition des Arts décoratifs à Paris (Blondel 1993 : 332). 
Fig.8. Sableuse Mangon, inspirée du principe du dépolissage par projection de sable sur le verre

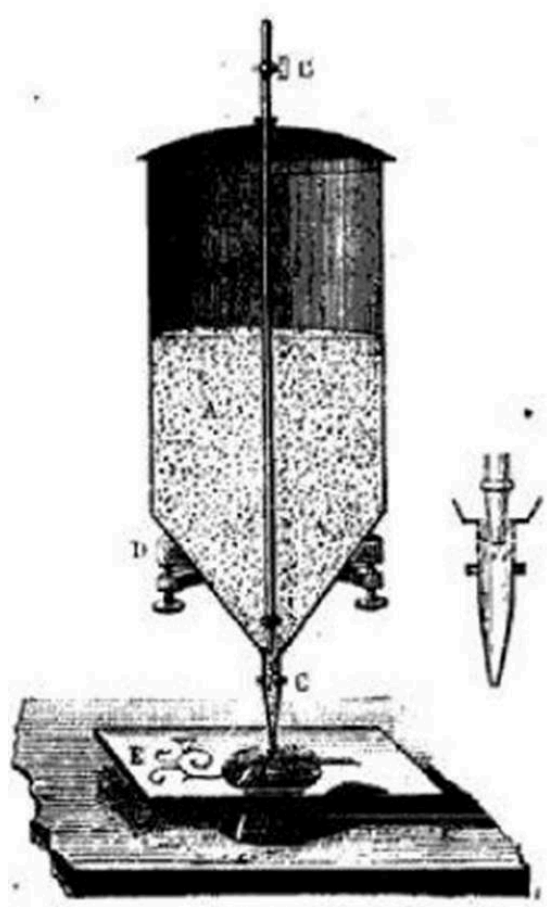

Fig. 10.

Description de la sableuse Mangon par Peligot : « [...] : c'est une trémie (fig.10) contenant du sable bien $\sec A$, qui s'écoule d'une manière continue par un tube $C$ dont on règle la longueur et l'inclinaison de manière à graduer à volonté la chute du sable : cet écoulement se fait par un tube étroit placé un peu au-dessous du tube qui amène le jet de vapeur ou le vent d'une machine soufflante. Des trous d'air comme dans les trompes, sont pratiqués à une petite distance du tube qui amène le vent. Le sable entrainé violemment par ce jet, est projeté avec force sur le corps E qu'on soumet à son action ».

PELIGOT, Le verre, son histoire, sa fabrication, 1877, p.71, BNF Gallica

\section{La Pinacophanie}

Cette technique apparaît vers le milieu du XIX ${ }^{\mathrm{e}}$ siècle. C'est un type d'ornementation en faux vitrail, qui vise particulièrement à imiter les verres mousseline en insérant un tissu peint entre deux feuilles de verre (Blondel 1993 : 333). Son concepteur, Eugène Debruel, peintre en bâtiments, enregistre son invention en 1848 : «Procédé propre à remplacer le verre dépoli, gravé ou peint» (Blondel 1993). Le tissu employé est généralement de la mousseline, ou une étoffe fine quelconque. Afin de la rendre complètement imperméable, Debruel l'apprête d'abord, en lui donnant deux à trois couches de cire vierge dissoute au bain-marie. Au bout d'environ 12 heures, lorsque la toile a totalement séché, il peint le motif au pinceau, avec des couleurs diaphanes ou du vernis. Le motif peut également être imprimé sur le tissu par les mêmes procédés employés pour l'impression sur étoffe. Au terme de ces étapes, la peinture est fixée par une couche de vernis incolore. L'étoffe ainsi peinte est placée entre deux verres ajustés aux bords par des bandes de toile collées, qui la préservent de l'humidité. Quinze ans après, en 1863, le peintre-verrier Victor-Julien Cassaignes brevète un "Système de peinture entre deux verres ${ }^{27}$. Il semble que ce brevet fasse suite à celui de Debruel, qui avait expiré la même année. 

sera complètement mis au point que dans les années 1890, avant de prendre son essor au $\mathrm{XX}^{\mathrm{e}}$ siècle et s'imposer par la suite dans la décoration sur verre.

CNUM, http://cnum.cnam.fr/

\section{BIBLIOGRAPHIE}

BARBÉ Jean-Julien, À travers le vieux Metz : Les Maisons historiques, t.I, Marseille, Laffitte reprints, 1976

BLONDEL Nicole, Le vitrail : vocabulaire, typologie et technique, Paris, Inventaire général, Imprimerie nationale, 1993 
CASSIDY Joseph, « More Than Scratching the Surface », The Pensylvania Center for the Book, http:// pabook.libraries.psu.edu/palitmap/Sandblasting.html, consulté le 10 octobre 2013

CLÉMANDOT Louis, « Verres colorés en feuilles ", Exposition universelle internationale de 1878 à Paris. Rapports du jury international. Groupe III - Classe 19, Rapport sur les cristaux, la verrerie et les vitraux, Paris, Imprimerie nationale, 1880

COMBES Charles, PELIGOT Eugène, « Séances du Conseil d'Administration, Procès-verbaux, Séance du 26 avril 1872 ", dans Bulletin de la Société d'Encouragement pour l'Industrie Nationale, $71^{\mathrm{e}}$ année, $2^{\mathrm{e}}$ série, t.19, Paris, Mme Veuve Bouchard-Huzard, 1872

EBELMEN Jacques-Joseph, « Peinture sur verre », Rapport du jury central sur les produits de l'agriculture et de l'industrie exposés en 1849. Vol. 2, Septième commission : Arts céramiques, t.II, Paris, Imprimerie nationale, 1850

GUGNON Louis-Napoléon, « Notices Industrielles extraites des publications Françaises et étrangères, Procédé de gravure sur verre ; par M. Gugnon à Metz », Bulletin de la Société d'Encouragement pour l'Industrie Nationale, $58^{\mathrm{e}}$ année, $2^{\mathrm{e}}$ série, t.6, Paris, Mme Veuve BouchardHuzard, 1859

LOZANO Fabiola, « Adolphe Lacroix: A Chemist and an Industrialist Popularizing Enamels in the Nineteenth Century », Architectural Glass Concepts Magazine, vol.5, 2012, https:// www.dropbox.com/s/e03vtkwn6lb4dy9/AGC\%20V5\%20ISSUE\%202_0212.pdf

LOZANO Fabiola « Le dilemme de la renaissance du vitrail au XIX ${ }^{\text {e }}$ siècle : entre redécouverte des techniques ancestrales et développement de techniques nouvelles ", Les innovations verrières et leur devenir, Actes du colloque organisé par l'Association Verre et Histoire, Nancy, 26-28 mars 2009, La Mothe-Achard (Vendée), Imprimerie Offset 5, 2013, http://www.verre-histoire.org/ colloques/innovations/pages/p403_01_lozano.html

LOZANO Fabiola, « Réapprendre le geste technique : la peinture sur verre en France au XIX ${ }^{\mathrm{e}}$ siècle In BOUILLON, Didier (dir.) et al., Gestes techniques, techniques du geste [en ligne]. Villeneuve d'Ascq : Presses universitaires du Septentrion, 2017. DOI : https://doi.org/10.4000/books.septentrion. 12372

PELIGOT Eugène, Le verre : son histoire, sa fabrication, Paris, G. Masson, 1877

SALVETAT Louis-Alphonse, « Rapport fait par M. Salvetat, au nom du comité des arts chimiques sur les Verres Mousselinés Colorés, présentés par M. Aubriot, fabricant rue du Faubourg SaintDenis, 190, à Paris ", Bulletin de la Société d'Encouragement pour l'Industrie Nationale, $76^{\mathrm{e}}$ année, $3^{\mathrm{e}}$ série, t.4, Paris, Société d'encouragement pour le développement de l'industrie nationale, 1877

\section{Webographie}

Archives INPI, http://bases-brevets19e.inpi.fr/

United States Patent and Trademark Office (USPTO), http://www.uspto.gov

Verrerie Mousseline.fr. Christian Fournié - Maître artisan verrier, http://verrerie-mousseline.fr

\section{NOTES}

1. Les techniques à chaud, à la différence de celles à froid, nécessitent une cuisson pour fixer les décors. 
2. DUVAL Charles, Brevet d'invention et de perfectionnement de 10 ans du 20 novembre 1837, Cat. 1838, p.236, cote : 1BA6292.

3. MARREL Jean-Pierre, Brevet de perfectionnement et d'addition du 31 décembre 1838, au brevet pris par Duval le 20 novembre 1837, Cat.1840, p.339, 1BA6292(1).

4. DUVAL Charles, Brevet d'invention de 15 ans du 5 janvier 1848, cote : 1BB6986

5. PAILLEUX Nicolas-Auguste, Certificat d'addition du 30 juin 1852, au brevet pris par Duval le 5 janvier 1848, cote 1BB6986(1).

6. DUMAS Joseph et GODARD Émile, Brevet du 11 juin 1841, Cat.1828-1842, p.562, cote 1BA9514.

7. Charles Paris (1823-1895), fils de Jean-Alexandre Paris, fondateur en 1827 de la cristallerie de Bercy. Charles succède à son père, invente en 1860 le verre opale au spath fluor et transfère au Bourget la verrerie de Bercy. ALPHANDÉRY M.F. Dictionnaire des inventeurs français, 1963, [Disponible sur World Biographical Information System (WBIS)]. http://db.saur.de/WBIS/login.jsf, consulté 1er septembre 2010.

8. GUGNON Louis-Napoléon, Brevet d'invention de 15 ans du 28 septembre 1861, cote 1BB51337; Brevet d'invention de 15 ans du 26 juillet 1865, cote : 1BB68215; Brevet d'invention de 15 ans du 23 mars 1866, cote 1BB70913.

9. BIEVEZ Désiré, Brevet d'invention de 15 ans du 17 février 1866, cote 1BB70280.

10. GUGNON, Brevet d'invention de 15 ans du 4 juillet 1865, cote 1BB67964; Brevet de 15 ans du 15 juin 1870, cote 1BB90386.

11. GUGNON Louis-Napoléon, Brevet d'invention du 17 octobre 1853, cat.1853, p.361, cote $1 \mathrm{BB} 17612$.

12. GUGNON, Mémoire descriptif du brevet, Op.cit., cote 1BB17612, p.3.

13. GUGNON Louis-Napoléon, Brevet d'invention de 15 ans du 3 septembre 1855, Cat.1855, p. 471 , cote 1 BB24619.

14. GUGNON Louis-Napoléon, Certificat d'addition du 3 mai 1856 au brevet pris le 3 septembre 1855, cote 1BB24619 (1).

15. TOURNEMIRE Guillaume, «Louis Napoléon Gugnon », Base collaborative Pierfit affilié à Geneanet, http://gw.geneanet.org/pierfit? lang=fr\&p=louis+napoleon\&n=gugnon, consulté le 7 février 2017.

16. AUBRIOT Édouard, Brevet d'invention de 15 ans du 29 décembre 1863, cote 1BB61298.

17. AUBRIOT, Brevet d'invention de 15 ans du 27 mars 1866, cote 1BB70998; Brevet de 15 ans du 13 mai 1874, cote 1BB103434.

18. DUPIEU, Brevet d'invention de cinq ans du 6 septembre 1808, (Expiré), Le dossier est porté manquant, cote 1BA1330; Brevet d'invention de cinq ans du 11 septembre 1813, cote 1BA465. t. VIII, n.606, p.39.

19. VERNERT Jean-François, Brevet d'invention de cinq ans du 10 décembre 1817, cat. 1791-1827, p.124, t.IX, p.338, cote 1BA1123.

20. FOURNIER François, Brevet d'invention de 15 ans du $1^{\text {er }}$ avril 1846, n.3244, p.307, cote 1BB3244.

21. GUGNON, Brevet d'invention de 15 ans du 8 août 1868, cote 1 BB110620.

22. TILGHMAN Benjamin, Brevet d'invention de 15 ans du 22 août 1870, cote 1BB90894. 
23. "Histoire de l'Aérogommage », http://www.aerogomm.com/accueil/histoire-de-laerogommage/, consulté le 10 octobre 2013.

24. Trompe : PHYS. Trompe à eau, à mercure. Appareil dans lequel un courant de liquide (eau, mercure) ou de vapeur (de mercure) permet de faire un vide partiel (Duval 1959). CNTRL http://www.cnrtl.fr/definition/trompe, consulté le 15 février 2017.

25. http://www.aerogomm.com/accueil/histoire-de-l-aerogommage/, op.cit., consulté le 10 octobre 2013.

26. http://www.aerogomm.com/accueil/histoire-de-l-aerogommage/, op.cit., consulté le 10 octobre 2013.

27. CASSAIGNES Victor-Julien, Brevet de 15 ans du 12 octobre 1863, cote 1BB60391.

28. ENGELMANN et GRAF, Brevet d'invention de 15 ans du 18 juillet 1856, cote 1BB28527, Bnf http://data.bnf.fr/12544505/eugene-melchior_peligot/.

\section{RÉSUMÉS}

$\mathrm{Au}$ cours du XIXe siècle, la renaissance du vitrail, puis son industrialisation et sa reconversion dans les arts décoratifs, ont favorisé le développement de nouveaux procédés de décoration sur verre, et l'apparition de nouveaux produits. Obtenu en imprimant des motifs répétitifs sur le verre à vitre ordinaire par dépolissage, le verre mousseline en est l'expression la plus représentative. Le verre dépoli perd sa transparence mais non sa translucidité, ce qui lui permet de voiler les intérieurs tout en laissant passer la lumière. Le charme du verre mousseline réside justement dans ce jeu entre transparence et translucidité qui compose son ornementation. Pour ceux qui n'ont pas les moyens de s'offrir un vitrail, c'est une alternative de décoration intéressante et abordable, et pour les architectes, une nouvelle option esthétique pour tamiser la lumière. La demande s'est donc envolée, dans la construction, pour la décoration de vestibules, salles de bain, vérandas et autres espaces auxquels on voulait donner un certain style. Face à la demande grandissante, les peintres-verriers et les décorateurs sur verre mirent au point des systèmes de production de verre mousseline plus performants, dont beaucoup firent l'objet de brevets d'invention. Par l'analyse de plusieurs de ces brevets, nous retraçons l'évolution des diverses techniques d'impression et de dépolissage du verre.

During the nineteenth century, a period rich in inventions and technical discoveries, the revival of stained glass followed by its industrialization and transformation into an applied art, led to the promotion and development of new glass decoration processes, which in turn led to new products. Among these novelties, muslin glass is the most representative expression. While domestic stained-glass was becoming fashionable, the question became how to find an inexpensive way to make ornamental glass within the reach of the public's budget. One of the solutions to this demand was to print repetitive patterns onto ordinary glass panes by frosting. Frosting consists in removing shine to make a surface matte. But, as far as glass is concerned, one does not simply remove shine, because frosting glass removes at the same time one of its main qualities: transparency. Though frosted glass loses its transparency, it does not lose its translucency, allowing it to veil what is inside from what is outside, while still letting light pass. The charm of muslin glass resided precisely in this game between the transparency and 
translucency which composed its ornamentation. For those who could not afford stained glass, frosted glass was an interesting and less expensive alternative, as well as representing for architects a new aesthetic option to sift light. Demand for frosted glass in buildings was rapidly increasing for the decoration of vestibules, bathrooms, verandas and other areas for which a particular style was desired. These panes were known as "frosted glass with eyelet drawings" or "muslin glass" or even "tulle glass". They were so called because those who conceived it sought to imitate embroidered curtains in muslin, tulle, or lace. Moreover, they often used this kind of textile as a stencil to decorate on the glass. Challenged with growing demand, glassmakerpainters and glass decorators developed more efficient production systems for muslin glass, many of which were the subject of patents. By analyzing several of these patents we will trace the evolution of the various techniques of printing and frosting glass.

\section{INDEX}

Mots-clés : histoire des techniques, verre, industrialisation, art industriel, ornementation, maison, dépolissage, innovation

Thèmes : Un objet une technique

Keywords : history of technology, glass, glassmaking, frosting, ornamentation, house, industrial art, industrialization

\section{AUTEUR}

\section{FABIOLA LOZANO}

Designer industriel de l'Universidad Nacional de Colombie en 1988, ses stages dans deux ateliers de vitrail parisiens à partir de 2001, où elle a expérimenté diverses techniques relatives au vitrail et à la décoration sur verre, ont fait d'elle une passionnée de cette matière. Docteur en Histoire des Techniques du CNAM, Paris, en 2013, avec une thèse intitulée « Innovations des techniques verrières au XIXe siècle et leurs applications dans la réalisation de vitraux ", elle combine la recherche historique et la création, toujours dans le domaine du verre. En 2019, elle installe son atelier de vitrail à Villedieu-les-Poêles en Normandie où elle travaille actuellement. 